\title{
Enlazando tradición e innovación. El programa integral de actuación para el centro histórico de asunción
}

\author{
Mabel Causarano ${ }^{1}$
}

Tipo de trabajo: Artículo

Material original autorizado para su primera publicación en el Journal de Ciencias Sociales de la Facultad de Ciencias Sociales de la Universidad de Palermo

\section{Recibido 27-8-2012}

Aceptado 30-9-2012

\section{Resumen}

Por casi tres décadas, el Centro Histórico de la ciudad de Asunción (CHA) ha sufrido un proceso de pérdida de la población residente y de centralidad urbana, causado por el desplazamiento de las actividades económicas y de las funciones residenciales hacia otras zonas de expansión metropolitana. Esta situación ha provocado su debilitamiento como escenario urbano y símbolo / fundacional de la nación. A estos fenómenos se agrega un nivel muy bajo de aprehensión del espacio público como lugar común, punto de encuentro, representación social y escenario de las relaciones culturales y cívicas. Con motivo del Bicentenario de la Independencia de España, el Gobierno de la República y la Municipalidad de Asunción, junto con diversos operadores privados, pusieron en marcha diversos proyectos e iniciativas, muchos de ellos focalizados en el CHA. El Programa Ciudadela Cultural de Asunción (PCCA), promovido y coordinado por la Secretaria Nacional de Cultura (SNC), plantea la recuperación del CHA desde la perspectiva de su dinamización como espacio cultural, conectándola a procesos de reactivación económica en los ámbitos del turismo, la economía creativa y la innovación. La cultura adquiere un valor estratégico: garantizar el carácter inclusivo en la recuperación del CHA, devolviendo al espacio público su condición de escenario de relación cívico y social, promoviendo la socialización de valores como la convivencia, el respeto, el concepto de bien común, la diversidad, la creatividad y la innovación. EI PCCA plantea actuaciones desde un enfoque y una metodología innovadores, basados en el rescate de la memoria colectiva y en la articulación entre instituciones públicas, organizaciones civiles y sector privado, para hacer del CHA un espacio urbano en el que la tradición y la creación se aúnen y promuevan una mejor calidad de vida y mayor cohesión social.

Palabras clave: Centro Histórico, recuperación urbana, articulación institucional.

\footnotetext{
${ }_{1}^{1}$ Arquitecta y urbanista, graduada y doctorada en la Universitá degli Studi di Roma, Italia. Docente e investigadora, con varios libros y artículos publicados. Actualmente es Ministra de la Secretaría Nacional de Cultura del Paraguay.

E-mail: mabelcausarano@gmail.com
} 


\section{TRADITION AND INNOVATION: THE INTEGRAL PROGRAM OF THE HISTORIC CENTER OF ASUNCION.}

\section{Abstract}

Over almost three decades, Asuncion's Historic Center (CHA) has undergone a gradual loss of its residents and of its urban centrality, due to the exodus of economic activity and residential settings towards other areas of metropolitan expansion. This situation has weakened the Center's role as urban scenery and as a foundational symbol for the nation.

Additionally, there is a lack of understanding of public spaces as common spaces; as places to come together; as social representations and centers of cultural and civic relations. On occasion of the Bicentennial of Paraguay's independence from Spain, the National Government and the City of Asuncion, together with a variety of private partners, began to unveil several projects and initiatives, many of them centered in the CHA. The "Ciudadela Cultural de Asunción" Program (PCCA) is promoted and coordinated by the National Secretariat of Culture (SNC). It proposes the recovery of the CHA by focusing on its dynamism as a cultural space, and connecting it to the revival of economic activities in areas such as tourism, creative economies and innovation. Through this work, culture attains strategic value: it guarantees inclusiveness in CHA's recovery, returning to the public space its quality of civic and social venue. This approach promotes the socialization of values such as coexistence, respect, the concept of public goods, as well as diversity, creativity and innovation. The Ciudadela Program proposes innovative approaches and methodologies, grounded in the retrieval of the collective memory and in the collaboration among public institutions, civil society organizations and the private sector. The goal is to turn CHA into an urban space where tradition and creation come together to promote a better quality of life and stronger social cohesion.

Key words: Historic Center, urban centrality, cultural policy

\section{Un marco propicio: el Bicentenario de la Independencia}

El 14 y 15 de mayo de 2011 el país se aprestaba a conmemorar los 200 años de la independencia de España. Desde la SNC se marcó una clara línea de trabajo. Sin obviar la organización de una serie de eventos festivos, las acciones apuntarían a la apertura de espacios desde los cuales estimular la memoria reflexiva y la construcción colectiva de una visión de futuro.

En una de sus intervenciones públicas, el Ministro Ticio Escobar (2009) expresó: "Más que eventos, el Bicentenario busca abrir una escena de acontecimientos; la posibilidad de que intelectuales, historiadores, políticos, artistas y ciudadanos en general piensen y reelaboren, desde distintos lugares, lo ocurrido desde mayo de 1811 hasta hoy." ${ }^{2}$

"Repensar la historia" - añadió - significa también cautelar el patrimonio, fortalecer las memorias colectivas e imaginar a través de la creación nuevas salidas para un tiempo difícil”, además de "impulsar la imaginación creadora a través de textos, figuras, música y representaciones capaces de recolocar los lugares de la memoria, bosquejar las líneas de un horizonte más claro y propiciar futuros mejorados" (Escobar, 2009).

\footnotetext{
${ }^{2}$ Tras la destitución del Presidente Fernando Lugo, el 22 de junio de 2012, el Ministro Ticio Escobar presentó renuncia al cargo.
} 
Constituida la Comisión Nacional del Bicentenario, a finales de 2009, se definieron como principales líneas de acción la protección y recuperación del patrimonio histórico, artístico, cultural, arquitectónico y edilicio; la reedición de obras clave para la interpretación de la historia paraguaya y el impulso a la producción de nuevas lecturas de una realidad compleja, que exige cruzar y confrontar diversos enfoques.

Tarea no fácil, por cierto, en un contexto en el cual la historia sigue siendo narrada como sucesión de hechos y gestas lideradas por protagonistas barnizados con pátinas que, según la óptica, los presentan como héroes o villanos; en el cual es arduo el esfuerzo para romper el maniqueísmo y el dogmatismo y así introducir miradas e interpretaciones plurales. Para muchos de quienes habitan el territorio paraguayo, la cultura es concebida como ornamento y privilegio de quienes disponen de tiempo y recursos materiales para acceder a ella.

La paraguaya, una de las sociedades más desiguales del mundo, es poco incline a la reflexión. Y, cabe señalarlo, enfrenta un lento y contradictorio proceso de reconstitución de la esfera pública, tras la larga dictadura de Stroessner, cuyos fantasmas reaparecen apenas el sistema democrático muestra signos de debilidad.

Origen del Programa Ciudadela Cultural de Asunción para la recuperación del Centro histórico de la Capital (PCCA)

El programa nació por iniciativa de la SNC, en articulación con otras quince instituciones públicas y privadas, con el propósito de impulsar la recuperación del carácter simbólico del CHA, desde la perspectiva de su dinamización como espacio cultural, conectándolo a procesos de reactivación económica, en los ámbitos del turismo y la innovación, la mejora de la calidad de vida y la cohesión social.

Arrancó formalmente en mayo de 2010, con la conformación de una alianza entre las instituciones que, con motivo del Bicentenario, tenían en sus carteras obras y un abanico de otros proyectos a ser ejecutados en el CHA, varias de las primeras de fuerte impacto urbanístico, social y ambiental, como el tramo inicial de la avenida Costanera Norte, dos parques costeros y la reconversión de la zona portuaria.

Estas intervenciones, emprendidas por el Ministerio de Obras Públicas y Comunicaciones (MOPC), fueron largamente postergadas ${ }^{3}$, con la progresiva disminución de la funcionalidad urbana, el deterioro de la cohesión social y la calidad de los recursos naturales; en particular, la Bahía de Asunción, colmatada por la erosión del suelo y el arrastre de residuos sólidos y contaminada por los aportes de efluentes de variado origen. La proximidad del Bicentenario generó un clima favorable para zanjar los obstáculos que, por rencillas y desentendimientos políticos, habían impedido por dos décadas la ejecución de estos importantes equipamientos urbanos.

La oportunidad comportaba la amenaza de que las obras privilegiaran los aspectos funcionales, a causa, en parte, de la estrechez de los plazos necesarios para ejecutarlas. La condición costera de Asunción, históricamente desaprovechada en sus potencialidades paisajísticas, culturales, ambientales, lúdicas y económicas, habría podido desplegarse si, a la necesaria atención a los

\footnotetext{
${ }^{3}$ El Plan Maestro de la Franja costera de Asunción fue elaborado en 1993. Desde entonces, ha tenido dos estudios de factibilidad y un préstamo del BID pronto para su desembolso, en 2004 , operación que fue abortada por desentendimientos entre el gobierno nacional y el municipal.
} 
aspectos funcionales del equipamiento vial, se integraban consideraciones que apuntaran a la valorización de las costas, el paisaje, el agua y los humedales, mejoraran la relación verde/ habitante, favorecieran el uso múltiple de los sistemas naturales y del espacio construido, con efectos positivos en la calidad de vida urbana, en general, y en las condiciones del CHA, en particular.

Entre las acciones proyectadas para conmemorar el bicentenario, la SNC tenía prevista la adquisición de edificios de valor histórico y arquitectónico ubicados en el CHA y la restauración de varios de ellos, que se sumarían a los que habría intervenido el MOPC. Se pensó integrar estas actuaciones en un programa ampliado que las complementara y sinergizara.

Hubo desafíos que encarar, entre ellos, el cambio de algunas actitudes predominantes, como las que sostenían las prácticas compartimentadas, con escasa o nula coordinación entre instituciones y hasta entre dependencias de una misma institución, la búsqueda de un enfoque común entre iniciativas nacidas, algunas de ellas, en momentos diferentes y con miradas sectoriales, la definición de una forma de trabajo que permitiera la articulación, sin que esta implicase una sobre exigencia para técnicos y funcionarios públicos muy demandados por sus propias rutinas.

\section{Una breve mirada retrospectiva del Centro Histórico de Asunción}

A partir de la década de los '80s, el CHA fue experimentando un proceso de deterioro físico y socioeconómico. A la par que se intensificó y extendió la conurbación metropolitana, en el área matriz disminuyó sensiblemente la función residencial, aumentó la informalidad y el stock edilicio fue perdiendo valor de mercado. En el 2008, cerca del $70 \%$ de los edificios en altura estaba parcialmente desocupado, diversos locales comerciales habían abandonado la zona y empeoraban las condiciones de los equipamientos públicos, en particular, las veredas y plazas.

El área matriz - que, al inicio, fue la ciudad misma y dos siglos y medio más tarde sede de la gesta independentista - surge como fuerte en 1537. A lo largo de la historia urbana se posicionó como el centro dinámico de la ciudad. Hasta hace 150 años, se confundía con la ciudad misma, por ser la parte edificada y con mayor densidad de ocupación del vasto territorio agrario circundante, bordeada por el río y las unidades productivas que abastecían el consumo urbano. La primera ampliación importante se dio recién a finales de 1800, con la llegada de grupos de inmigrantes europeos y la conformación de nuevos barrios (Causarano, 2012)

El cambio socio demográfico reforzó la centralidad del CHA y su importancia política y económica, pues alojaba a las principales instituciones públicas, religiosas y educativas, a los bancos y empresas comerciales; allí se ubicaron las residencias de los sectores de clase alta y media; sus plazas eran las más concurridas y sus casas comerciales las más renombradas. La polifuncionalidad y el dinamismo social, económico y cultural se mantuvieron hasta inicios de los '70s, cuando la represión política, implantada por el régimen dictatorial del Gral. Stroessner, a partir de 1954, impidió el uso libre del espacio público, mediante diversas medidas coercitivas.

En esa misma década se instaló un proceso de modernización física de la ciudad, que implicó un drástico cambio en la imagen urbana y afectó fuertemente al CHA, como consecuencia del ingreso de capitales provenientes de la construcción de la represa binacional de Itaipu. 
El gobierno aplicó dos estrategias (Causarano, 2012):

a. Trasladar varios de los grandes equipamientos del $\mathrm{CHA}$ a los límites de la ciudad (como las sedes de algunas instituciones públicas, la Terminal de ómnibus, el Banco Central, el Palacio de Justicia, la Municipalidad, entre otros), con lo cual sustrajo al área matriz funciones y usuarios;

b. Densificar el área central, mediante la construcción de edificios en altura, destinados principalmente a oficinas de bancos y empresas financieras, sedes de organismos internacionales, embajadas y consulados, los cuales, junto con el fortalecimiento de la función comercial y de servicios - en detrimento de la residencial -, especializó la centralidad del CHA en el sector terciario.

La función residencial emigró a zonas de la ciudad hasta entonces poco o no urbanizadas, que fueron "polarizadas" por los nuevos equipamientos institucionales, creándose lo que se denominaría "nuevas centralidades", que recibieron residentes de sectores altos y medio - altos, mientras que los de menor poder adquisitivo se instalaron en los municipios cercanos a la capital, extendiéndose, por tanto, la conurbación metropolitana.

El fenómeno se intensificó en los años siguientes y comportó la disminución de la tasa de crecimiento demográfico de Asunción - que hoy está cercana al $0 \%$ anual - y la expansión del área metropolitana, inducida por las empresas inmobiliarias que ofrecen lotes en tierras cada vez más distantes de los lugares de trabajo de los compradores y aumentan la población de las ciudades dormitorio, algunas de las cuales ha visto crecer su población tasas del 10\% anual.

Entre los '70s y los ' 80 s, desaparecieron numerosos edificios de valor histórico y arquitectónico, para dar lugar a nuevas construcciones o para utilizar el predio como área destinada al estacionamiento de vehículos. Desde mediados de los '90s, las dinámicas sociales, culturales y económicas asuncenas fueron definidas por el mercado y la empresa privada: los centros comerciales y los conjuntos habitacionales cerrados connotaron las zonas de mayor inversión inmobiliaria y comercial. Se fortalecieron las nuevas centralidades y se postergó la recuperación y calificación del espacio público, con lo cual se aceleró el proceso de fragmentación social.

La apertura política y la adquisición de libertades no se tradujeron en la democratización de la ciudad, en cuanto bien público. Por el contrario, hubo una clara y sostenida opción por la mercantilización del desarrollo urbano, el aumento de la privatización de los lugares de entretenimiento y la progresiva disminución de la calidad de las áreas de uso colectivo, principalmente, las del $\mathrm{CHA}$ (calles, plazas, parques, costas del río).

La contracción de la intervención y la inversión públicas hizo que la ciudad fuera, como lo expresa Carrión (2000), escenario de una de las contradicciones principales de los centros históricos: la que se plantea "entre riqueza histórica - cultural y pobreza económica - social". En consecuencia, fue perdiendo su condición de espacio público, a la par que se fortalecía la exclusión social.

A pesar de ello, el CHA aún reúne las condiciones para localizar los recursos de identidad e innovación, mediante políticas que articulen cultura, cohesión social, medio ambiente y tecnología. No se trata de replicar "modelos exitosos" sino de ubicar el proyecto de recuperación en su contexto y con visión de futuro: asumir el enfoque que propicie la construcción de una "ciudad inclusiva", en donde todos puedan participar, productiva y positivamente, "en las oportunidades que la ciudad tiene para ofrecer", como plantea la Declaración de Estambul sobre los Asentamientos Humanos (1996). Una ciudad justa, que potencie la heterogeneidad sociocultural, en donde las personas "sin discriminaciones de género, edad, condiciones de salud, ingresos, nacionalidad, etnia, condición 
migratoria, orientación política, religiosa o sexual" tengan derecho a preservar la memoria y la identidad cultural (...)". (Carta Mundial por el Derecho a la Ciudad, 2005, Art. I).

\section{Oportunidad inédita}

En la SNC se comprendió que el Bicentenario habría podido ofrecer una oportunidad inédita para impulsar la recuperación del CHA, planteándola como una estrategia de desarrollo urbano, de fortalecimiento de la capitalidad asuncena, de convergencia programática entre el gobierno central y el gobierno local y de interacción público - privada, abriendo así la posibilidad de encarar las intervenciones con sentido de futuro.

El CHA es un lugar de diversidad, con potencial para promover la inclusión y convertirse en espacio de redes, pues mantiene polos de atracción (el Palacio de Gobierno, el Parlamento, la mayor parte de los ministerios, numerosos edificios emblemáticos y equipamientos culturales), concentra los sitios con la mayor carga simbólica del país, en él se despliega una importante cantidad de flujos (tránsito de vehículos, personas, actividades comerciales, educativas, religiosas), contiene una alta dotación de espacios públicos, la mayor parte del patrimonio monumental y puede aumentar sensiblemente la oferta de actividades culturales.

\section{Enfoque, metodología y procesos}

La articulación entre las instituciones que habrían conformado la alianza requirió acuerdos básicos sobre el enfoque y la metodología a utilizarse.

Se convino que, en el marco del Bicentenario, la cultura habría tenido un rol catalizador, no en el sentido eventista, folclorista o de suma de actividades lúdicas y de entretenimiento, sino como motor del desarrollo urbano, a partir del rescate de los factores históricos e identitarios que enlazaran la memoria y la visión de futuro para la ciudad. Junto con la protección y recuperación del patrimonio histórico, artístico, cultural, arquitectónico y edilicio, la acción en el CHA se prestaba a una re-lectura y a la producción de nuevas interpretaciones de la historia urbana.

La palabra Paraguay, pronunciada en idioma guaraní, con la "y" con sonido gutural, es el nombre de Asunción en la lengua nativa. Paraguay se denominó, hasta finales del 1700, el vasto territorio conocido hoy como Cuenca del Plata, dentro del cual el CHA ocupa una posición física central, así como fue y sigue siendo central en la dimensión lingüística, en su carácter de centro de la capital del único país bilingüe de la región y referente para la vasta familia guaranítica, que ocupa territorios en Argentina, Bolivia y Brasil. La centralidad cultural del CHA se recupera a partir del nombre del país y de la ciudad.

En guaraní, "y" significa agua. El CHA se ubica en el punto medio del eje fluvial del sistema hidrográfico Paraguay - Paraná. Expresada en el guaraní, la palabra "agua", además de lo que denota objetivamente, connota una centralidad cultural, la presencia y el valor del vital elemento, la condición costera y portuaria de Asunción, entre otros. Tiene un fuerte poder de evocación y, a la vez, desafía a la imaginación en términos de futuro, con el agua como fuente de vida (también como origen del territorio en época precolombina y posteriormente), recurso estratégico de uso múltiple, que induce la recuperación y puesta en valor de las costas, del sistema fluvial, de las actividades que 
tuvieron fuerte dinamismo y se fueron debilitando, como el transporte fluvial y los deportes náuticos, las ofertas para uso abierto y colectivo del agua y las costas.

A ello se suma la centralidad histórica, reflejada en la denominación "Madre de ciudades", calificativo que distingue a Asunción, pues, durante el siglo XVII, partieron desde ella las expediciones que fundaron varias de las actuales pujantes ciudades del Cono Sur americano.

El enfoque, por tanto, habría sido aquél que permitiera la recuperación integral de la condición costera de la ciudad, tomando como punto de apalancamiento, la valorización de las centralidad del CHA, a partir de la convergencia de actuaciones culturales, medioambientales, urbanísticas y de impulso a la economía creativa, en el marco de la gobernanza territorial. El PCCA planteó que las intervenciones tuvieran sentido de futuro y no de "reconstrucción del pasado", aseguraran el cumplimiento del derecho a la ciudad, priorizaran y re - significaran lo público como opción frente a los problemas urbanos, fortalecieran el poder de convocatoria del $\mathrm{CHA}$ y lograran que toda la ciudad se reconozca en él.

Al ser pensado e intervenido por una variedad de sujetos (instituciones públicas, organizaciones privadas e individuos), el CHA habría adquirido una nueva centralidad: la que le confiere el ser objeto de actuaciones y proyectos que condensan anhelos, expectativas e intenciones de saldar algunas de las deudas históricas de la capital con la ciudadanía y de los gobiernos nacional y municipal con el CHA.

No hubo mayor dificultad para articular los proyectos desde este enfoque, que fue plasmado en el Programa Integral de Actuación (PIA), lográndose que la cultura, en su pluralidad de facetas y expresiones, constituyera el adhesivo que uniera las acciones que habrían convergido en el $\mathrm{CHA}$. Así, la Avenida Costanera es mucho más que el necesario canal de tránsito que bordea la costa y pasa a actuar como eje paisajístico de enlace entre la ciudad y el agua, generador de oportunidades para el desarrollo del comercio, la vivienda, el turismo, las ofertas de entretenimiento, de nuevos espacios abiertos, de eventos educativos y culturales.

El enfoque, que plantea el rescate de las memorias en función del futuro deseable para la comunidad concreta, es aplicable a cualquier lugar en donde se active el proceso de recuperación y puesta valor del territorio.

La replicabilidad se asegura al valorizar los aspectos identitarios y la memoria colectiva del lugar concreto. Cada sitio tiene historia, acumula y sedimenta experiencias, vive el presente y encierra posibilidades de futuro.

La articulación se basó en una metodología que ofrece flexibilidad y eficiencia para la labor conjunta de dieciséis instituciones, entre ministerios y secretarías de estado, la municipalidad de Asunción, la Universidad Católica y la Universidad Nacional, organizaciones comunitarias y asociaciones de empresarios ${ }^{4}$. Se plasmó una alianza, mediante la cual las acciones se irían desarrollando en dos niveles: el estratégico y el operativo.

El primero, se organiza en torno a la Mesa Articuladora interinstitucional (MAI), formada por las máximas autoridades de las instituciones aliadas o por sus representantes, al cual cabe definir políticas, estrategias y facilitar la gestión. El segundo, corresponde al Equipo Técnico Interdisciplinario (ETI), que reúne a expertos nacionales y trabaja en conjunto con los asesores

\footnotetext{
${ }^{4}$ La Mesa Articuladora Interinstitucional está formada por representantes del Ministerio de Obras Públicas y Comunicaciones, la Municipalidad de Asunción, el Ministerio de Hacienda, la Secretaría Nacional de Turismo, la Secretaría del Ambiente, la Comisión Nacional del Bicentenario y la Universidad Católica, entre otras.
} 
externos ${ }^{5}$.

Desde el inicio, el sector privado participó en distintas actuaciones, a través de organizaciones gremiales y sociales ${ }^{6}$, en la elaboración del análisis y el diagnóstico del $\mathrm{CHA}$ y, posteriormente, en el proceso de discusión y aplicación del PIA. ${ }^{7}$

Se promovió, desplegó y valorizó la capacidad local. Los técnicos nacionales, designados por sus respectivas instituciones, contribuyeron, desde sus campos de conocimiento disciplinario y su experiencia en la gestión, a la comprensión de las potencialidades del CHA, a la definición del menú de actuaciones y al análisis de su factibilidad. El trabajo compartido con la asesoría externa fue altamente efectivo, porque permitió la transferencia tecnológica en materia de planificación urbana. La misma se dio en un doble sentido: desde el equipo externo hacia el local y desde este hacia los asesores, quienes pudieron aprehender en la práctica las dinámicas urbanas, sociales, económicas e institucionales de la ciudad y el país, lo cual "endogeneizó" la contribución externa.

La metodología, que también facilita la replicabilidad, al involucrar a los actores clave de cada realidad territorial, incluye la capacitación continua de los técnicos que participan en el proceso y la formación de nuevos talentos que se incorporan a los cursos y seminarios, de acuerdo con las exigencias de un proceso de fuerte dinamismo y a las oportunidades de revalorizar otras áreas de la ciudad y las de otros municipios ${ }^{8}$.

\section{El desarrollo del PIA y la ejecución de las actuaciones}

EI PIA es un instrumento pensado para articular y priorizar acciones y proyectos, facilitar la gestión, en el marco de una estrategia que, desde la perspectiva de la cultura y en coherencia con los objetivos comunes de otros proyectos en marcha, movilice recursos públicos y privados. Contempla tanto la estrategia general para su desarrollo, como una fórmula organizativa para su funcionamiento operativo y relacional.

EI PIA se organiza en cinco ejes, con acciones que, si bien se adscriben a uno de ellos, tienen componentes que contemplan a los demás:

- $\quad$ Impulso y fortalecimiento integral de la dimensión cultural;

- $\quad$ Impulso y fortalecimiento del ocio y el turismo local e internacional;

- Impulso de la economía creativa, que sinergiza la creatividad, la cultura, la economía y la tecnología, a la vez de generar vínculos, a nivel macro y micro, entre las varias facetas de la economía;

- $\quad$ Mejora del espacio público y del tejido institucional;

- $\quad$ Proyección nacional e internacional del CHA, como estrategia de desarrollo de la calidad de vida.

Se apunta a transformar Asunción en una ciudad innovadora, con una red de iniciativas públicas, cívicas y empresariales que extraigan y pongan en valor el conocimiento; una ciudad conectada al mundo, que promueva el aprendizaje mediante corredores culturales, participativos,

\footnotetext{
${ }^{5}$ La asesoría externa estuvo a cargo de la Fundación Barcelona Media, a través de su Laboratorio de Cultura y Turismo, dirigido por Jordi Pardo. www.barcelonamedia.org.

${ }^{6}$ Entre ellas, la Asociación de Empresarios del Centro, la Asociación de Hoteleros del Paraguay y la Cooperativa Ricardo Brugada, que congrega a los pobladores de un barrio popular, La Chacarita, que forma parte del CHA.

${ }^{7}$ Fue presentando al Presidente de la República el 07 de noviembre de 2010, en un acto de carácter público.

${ }^{8}$ La Fase preparatoria, insumió 2 meses; la Fase analítica y diagnóstica, 5 meses. La fase ejecutiva inició en noviembre de 2010.
} 
tecnológicos y ambientales, estimule la habilidad para crear y distribuir capital intelectual, con potencial para generar ingresos, empleo, promover la inclusión social, la diversidad cultural y el desarrollo humano.

Entre las actuaciones, se prevé la creación del "Centro de la Memoria y la Innovación”, iniciativa que hará del CHA un nodo tecnológico y espacio de creación, activo y productivo, residencial y monumental, de convivencia de los sectores tradicionales con los nuevos ámbitos y actores sociales y económicos. Un lugar para presentar la ciudad y el país, su pasado y sus proyecciones.

Se fortalecerá el poder de convocatoria del CHA, para que la ciudadanía se reconozca en él; será un espacio de oportunidad e innovación, que conserva el patrimonio heredado gracias a la incorporación de los usos contemporáneos de la "ciudad del conocimiento": las redes institucionales, las viviendas, los centros culturales y de información, los equipamientos y espacios públicos, los acontecimientos y exposiciones, los proyectos de regeneración urbana.

\section{Acciones en marcha y aprendizajes compartidos}

La conmemoración del Bicentenario convocó a la ciudadanía de todo el país y tuvo en el CHA el epicentro de las celebraciones. Durante varios días, las calles, plazas y edificios de uso público atrajeron a una pluralidad de usuarios, la mayoría de los cuales no acudía habitualmente al área central. Para buena parte de ellos, fue un re - descubrimiento y, en algunos casos, la primera oportunidad de acercarse a la costa de la Bahía de Asunción, sin obstáculos físicos ni temores causados por la percepción de inseguridad. Según registros policiales, durante los días festivos, disminuyeron sensiblemente las denuncias de robos y asaltos.

A la restauración y puesta en valor de edificios emblemáticos, la construcción de dos parques costeros y del primer tramo de la avenida costanera, el PCCA va sumando acciones de movilización ciudadana que capitalizan la experiencia de apropiación de los espacios públicos y asumen diferentes modalidades: circuitos temáticos que incluyen a dos barrios populares, cuyos guías son vecinos capacitados para ofrecer el servicio; la apertura de la zona portuaria para un amplio menú de actividades que reúnen a niños, jóvenes, adultos y adultos mayores en torno a ferias, danzas, obras teatrales, conciertos, talleres didácticos, muestras, paseos en barco, gratuitas la mayoría de ellas y a precios muy accesibles las demás.

En la brevedad, se inaugurará el Centro de la Memoria y la Innovación, un espacio híbrido, para el fomento de las industrias culturales y creativas, la presentación de la ciudad y del país a través de la utilización de recursos digitales amigables, accesibles a todo público.

Uno de los proyectos clave es la creación de una agencia de gestión público - privada que se encuentra en formación. En una primera fase, la misma adoptará la figura de una asociación civil con capacidad restringida, denominada Asociación Ciudadela, debido a que la legislación paraguaya exige una ley especial para que el sector público y el privado conformen una sociedad ${ }^{9}$.

La experiencia ha arrojado aprendizajes importantes, entre los cuales, se destaca el valor de la acción conjunta e intersectorial, que permitió la continuidad del proceso, varias veces amenazada por los cambios de las autoridades de algunas de las instituciones públicas aliadas. En un contexto de bajo desarrollo institucional, buena parte de la ejecución de las políticas públicas depende de las ${ }^{9}$ La Asociación Ciudadela fue creada a mediados de julio de 2012. Forman parte de la comisión directiva exponentes del sector público, académico y empresarial. 
personas que están a cargo; son harto frecuentes las interrupciones de los procesos no sólo cuando cambia el gobierno nacional o municipal, sino cuando, en el período de un mismo gobierno, cambian los responsables.

La participación ciudadana en las actividades públicas que se realizan semanalmente desde hace año y medio, ha dado visibilidad al PCCA y constituido una demanda que va adquiriendo fuerza, creando hábitos, recuperando el poder de convocatoria del espacio público e instalándose en la agenda de los tomadores de decisión, lo cual le otorga sostenibilidad política, aspecto que fue detectado como una de las mayores amenazas en el análisis estratégico realizado a inicios del proceso de planificación. A tal efecto, la Asociación Ciudadela es una aliada relevante, que reúne a exponentes representativos de la institucionalidad pública y de los sectores más dinámicos del ámbito urbano.

EI PCCA se encuentra en un momento de consolidación, a pesar del impacto provocado por el abrupto e inesperado cambio de gobierno y la polarización social y política resultante. Es un logro que puede atribuirse a la paciente construcción de una "arquitectura de proceso", hecha a medida de las demandas y ofertas locales, con oportunas inyecciones de la experiencia externa.

\section{Referencias bibliográficas}

Achilli, E., Bernardi G., Calligaro, E., Cámpora E., de Castro, R., Giampani, L., Nemcovsky, M., Sánchez, S., Shapiro, J. (Coords.) (2005). Vivir la ciudad. Espacios Urbanos en disputa. Rosario: Laborde Libros Editor.

Aymonino, C. (1981). El significado de las ciudades. Madrid: H. Blume Ediciones

Bauman, Z. (2009). Modernidad líquida. Buenos Aires: Fondo de Cultura Económica.

Carrión M., F. (2000). Lugares o flujos centrales: los centros históricos urbanos. Serie Medio ambiente y Desarrollo, 29: 7.

Causarano, M. (2012). Cambios en el carácter público y la centralidad del Centro histórico. En Flores, A. (Coord.) Paraguay: una perspectiva. Las centralidades actuales y las posibles. Quito: OLACCHI. pp. 87 - 88 .

Coppola Pignatelli, P. (1992). L'identitá come proceso. Cultura spaziale e progetto di architettura. Roma: Officina Edizioni

Delgado, M. (2010). La ciudad mentiros. Fraude y miseria del "modelo Barcelona". Madrid: Catarata

De Oliveria, D. (2001). Curitiba e o mito da ciudades modelo. Curitiba: Editora UFPR

Escobar, T. (2009). Memorias colectivas y pensar el futuro. Revista del Bicentenario 1: 13. 
Hannerz, U. (1986). Exploración de la ciudad. México D:F.: Fondo de Cultura Económica

Krucken, L. (2009). Design e territorio. Valorizaçao de identidades e produtos locais. Sao Paulo: Studio Nobel e SEBRAE.

Moix, LI. (2010). Arquitectura milagrosa. Hazañas de los arquitectos estrella en la España del Guggenheim. Barcelona: Anagrama

Muñoz, F. (2010). Urbanalización. Paisajes comunes, lugares globales. Barcelona: Editorial Gustavo Gili

Proença Leite, R. (2007). Contra - usos da cidade. Lugares de espaço público na experiencia urbana contemporánea. $2^{\mathrm{a}}$ Edición. Campinas: Editora UNICAMP e Editora UFS

Puig, T. (2009) Marca ciudad. Cómo rediseñarla para asegurar un futuro espléndido para todos. Buenos Aires: Paidós

de Souza Araújo, R. (2010). Modificaçoes no planejamento urbanístico. Teoria e método de análise. Sao Paulo: Nobel

Szécsi, A. (2006). Reciclado de ciudades. Nuevas herramientos de planificación y diseño urbano para intervenir en ciudades existentes. Caso Buenos Aires. Buenos Aires: Nobuko

Ultramari, C. (2005). O fim das utopias urbanas. Sao Paulo: Studio Nobel

Viladevall i Guasch, Castrillo Romón, M. (Coords.) (2010). Espacio público en la ciudad contemporánea. Perspectivas críticas sobre su gestión, su patrimonialización y su proyecto. Valladolid: Universidad de Valladolid

\section{Documentos}

Carta Mundial por el Derecho a la Ciudad (2005)

Declaración de Estambul sobre los Asentamientos Humanos (1996)

Programa Integral de Actuación de Ciudadela Cultural de Asunción (2010) www.ciudadela.org. 